\title{
Post-Translational Regulation of miRNA Pathway Components, AG01 and HYL1, in Plants
}

\author{
Seok Keun Cho ${ }^{1}$, Moon Young Ryu ${ }^{1}$, Pratik Shah ${ }^{3}$, Christian Peter Poulsen ${ }^{4}$, and Seong Wook Yang ${ }^{1,2, *}$
}

\begin{abstract}
Post-translational modifications (PTMs) of proteins are essential to increase the functional diversity of the proteome. By adding chemical groups to proteins, or degrading entire proteins by phosphorylation, glycosylation, ubiquitination, neddylation, acetylation, lipidation, and proteolysis, the complexity of the proteome increases, and this then influences most biological processes. Although small RNAs are crucial regulatory elements for gene expression in most eukaryotes, PTMs of small RNA microprocessor and RNA silencing components have not been extensively investigated in plants. To date, several studies have shown that the proteolytic regulation of AGOs is important for host-pathogen interactions. DRB4 is regulated by the ubiquitin-proteasome system, and the degradation of HYL1 is modulated by a de-etiolation repressor, COP1, and an unknown cytoplasmic protease. Here, we discuss current findings on the PTMs of microprocessor and RNA silencing components in plants.
\end{abstract}

\section{INTRODUCTION}

MicroRNAs (miRNAs) are a class of regulatory small RNAs that control diverse biological events in plants, animals, and many single-cell eukaryotes (Baulcombe, 2004; Brodersen and Voinnet, 2009). By degrading or blocking the translation of target messenger RNAs, miRNAs modulate the expression of genes for development, differentiation, metabolism, and defense (Bartel, 2004; Voinnet, 2005; 2009). Because of the important roles of miRNAs in biological events, extensive researches have been performed to delineate the precise mechanisms of miRNA processing (Kim, 2005; Kurihara et al., 2006; Parizotto et al., 2004; Ren and Yu, 2012). In general, miRNAs are processed

${ }^{1}$ Department of Systems Biology, College of Life Science and Biotechnology, Yonsei University 03722, Korea, ${ }^{2}$ Department of Plant and Environmental Sciences, Center for UNIK Synthetic Biology, Faculty of Science, University of Copenhagen, Thorvaldsensvej 40, DK-1871 Frederiksberg, Denmark, ${ }^{3}$ Department of Biomedical Engineering, University of California Irvine, 92697, CA, USA, ${ }^{4}$ Carlsberg Research Laboratory, GI. Carlsberg Vej 4, 1799 Copenhagen V, Denmark

*Correspondence: yangsw@yonsei.ac.kr

Received 4 April, 2016; revised 9 June, 2016; accepted 10 June, 2016; published online 20 July, 2016

Keywords: AGO1, autophagy, HYL1, miRNA, protease, proteolysis, UPS from long transcripts, called primary miRNAs (pri-miRNAs), which are generated from their own genes or from introns (Okamura et al., 2007). A single pri-miRNA contains one or more imperfect hairpin-loop structure with miRNA sequences (Bielewicz et al., 2013; Meng et al., 2013). In animals, the apical element and stem of a pri-miRNA is recognized by DiGeorge Syndrome Critical Region 8 (DGCR8) through its doublestranded RNA domain and RNA-binding heme domain, respectively (Landthaler et al., 2004; Nguyen et al., 2015). Drosha, a type III RNAse, forms a heterotrimeric complex, known as a microprocessor, with two molecules of DGCR8 in order to process pri-miRNA into precursor-miRNA (pre-mRNA) (Gu et al., 2012; Kwon et al., 2016). The processed product is subsequently exported to the cytoplasm where it is further cleaved by another RNase type III enzyme, Dicer. Dicer recognizes $5^{\prime}$ and $3^{\prime}$ ends of the pre-miRNA and excises the loop to release mature miRNA (Denli et al., 2004; Saito et al., 2005; Zeng et al., 2005). In plants, the two-step cleavage of pri-miRNA is performed by a Dicer homolog, Dicer-like1 (DCL1). Two DCL1-binding proteins, HYPONASTIC LEAVES 1 (HYL1) and SERRATE (SE) are essential for the accuracy of pri-miRNA processing (Dong et al., 2008; Kurihara et al., 2006; Yang et al., 2010). HUA ENHANCER 1 (HEN1) adds a methyl group to the 3 ' end of miRNAs prior to their transport out of the nucleus (Park et al., 2002). In addition to these core proteins, more than 20 components are directly or indirectly involved in plant pri-miRNA processing (Ben Chaabane et al., 2013; Zhang et al., 2013; 2014). For instance, TOUGH (TGH) is a G-patch domain protein, which directly binds to the DCL1, SE, and HYL1 complex to assist in the recruitment of pri-miRNAs (Rogers and Chen, 2013). RECEPTOR FOR ACTIVATED C KINASE 1 (RACK1) is a scaffold protein required for protein-protein interactions, which interacts with SE to modulate pri-miRNA processing (Speth et al., 2013). SICKLE (SIC), a proline-rich protein, is involved in the maturation of miRNAs (Zhan et al., 2012). STABILIZED 1 (STA1), a pre-mRNA processing factor 6 homolog protein, regulates the splicing of pri-miRNAs (Ben Chaabane et al., 2013). The final step in both plants and animals involves mature miRNAs being selectively loaded to ARGONAUTE 1 (AGO1) protein, which is one of the essential catalytic components of the RNA-induced silencing complex (RISC) in the cytoplasm (Rogers and Chen, 2013).

Since the discovery of miRNAs and their roles in gene expression, most studies on miRNAs have focused on improving understanding of processing and functional mechanisms. In contrast to the post-translational modifications of proteins in many regulatory pathways, such as light signaling, hormone 
regulation, and stress responses, which have been widely studied in plants, knowledge on post-translational modifications of the miRNA processing and silencing machinery is still rudimentary. Recently, several pioneering studies on the posttranslational regulation of RNA silencing machinery have been performed in both animals and plants. This review focuses on published studies on the post-translational regulation of the silencing machinery, focusing on HYL1, DRB4, and AGO1 in plants. The biological impacts of proteolytic regulation will be discussed along with several perspectives on the current findings in this field.

\section{POST-TRANSLATIONAL REGULATION OF AGO1}

AGO1 plays key roles in both miRNA- and siRNA-mediated gene silencing either by destabilization of target mRNAs or by translational repression (Valencia-Sanchez et al., 2006). Considering the important functions of AGO1 in plant growth, development, and defense, it is not surprising that the expression of AGO1 is tightly regulated by highly programmed regulatory consoles (Bohmert et al., 1998). AGO1 is negatively regulated by miR168 and by itself, in a process known as AGO1catalyzed mRNA cleavage. In the self-regulation pathway, the transcriptional co-regulation of MIR168 and AGO1, and posttranscriptional stabilization of miR168 by AGO1, are important to modulate the efficiency of assembling RISC complexes (Vaucheret et al., 2004; 2006). In addition to post-transcriptional regulation, post-translational regulation of AGO1 protein has been reported in the context of plant-virus interactions (Alvarado and Scholthof, 2011).

Since the P0 protein of beet western yellows virus (BWYV) was shown to suppress the plant's RNA silencing defense mechanism. Many P0 proteins from members of the genera Polerovirus and Enamovirus have also been identified as suppressors (Pfeffer et al., 2002). Because of the F-box like central region, the $\mathrm{PO}$ proteins of these viruses are considered to be $\mathrm{F}$ box like proteins. In general, F-box proteins are part of the SCF SKp1-Cullin-F-box protein (SCF) E3 ubiquitin ligase complexes, which tether poly-ubiquitin to a target protein and subsequently mediate the modified protein for proteasome-mediated degradation (Callis, 2014; Cardozo and Pagano, 2004; von Arnim, 2001). In plants, the assembling of multiple F-box proteins into the same SCF complex permits the recognition of a broad range of target proteins (Hare et al., 2003). Therefore, it was suggested that the viral F-box protein (P0) proteins could snatch the host SCF ubiquitin-protein ligase (E3) system by mimicking the host F-box protein and subsequently incapacitating the host defense system. The hijacking ability of P0s has been reported in two poleroviruses, BWYV and Cucurbit aphidborne yellows virus (CABYV), which directly interact with S-phase kinase related protein 2 (SKP2), a component of the SCF family of ubiquitin E3 ligases (Pazhouhandeh et al., 2006; Pfeffer et al., 2002). However, this is insufficient to explain the exact mode of action of PO in impeding the host RNA silencing system.

In 2007, using the tobacco transient-expression system, Baumberger et al. showed that the $\mathrm{P} 0$ of Polerovirus targets the PAZ domain of AGO1 and leads to the destabilization of AGO1 (Baumberger et al., 2007). At the same time, Bortolamiol et al. (2007) also demonstrated that the ectopic expression of P0 in Arabidopsis triggers AGO1 degradation. Those authors further showed that $\mathrm{PO}$ is incapable of promoting the destabilization of pre-loaded AGO1. However, there is no clear evidence of a direct association between P0 and AGO1. This strategy, utilizing viral suppressor proteins to neutralize the AGO1-dependent defense system, is found widely in other viruses. P25 is the silencing suppressor of Potato virus $X$, which directly interacts with AGO1 (Chiu et al., 2010). Pea enation mosaic virus-1 (PEMV-1) is able to inhibit local and systemic RNA silencing systems by destabilizing AGO1 (Fusaro et al., 2012). Cucumber mosaic virus (CMV)-encoded $2 b$ protein directly interacts with AGO1 and inhibits the RNA silencing system, but is not associated with the destabilization of AGO1 (Chiu et al., 2010; Zhang et al., 2006).

Several studies have further demonstrated the detailed mechanism of AGO1 destabilization in both viral- and non-viral contexts. P0 of Polerovirus is known to deceive the host SCFtype complexes, resulting in the proteasomal degradation of their target proteins. Contrary to the relationship between P0 and SCF-complexes, the degradation of AGO1 by P0 was surprisingly unaffected by inhibition of the $26 \mathrm{~S}$ proteasome (Derrien et al., 2012). AGO1 was also negatively modulated by a host F-box protein, F-box, and WD-repeat domain-containing protein 2 (FBW2), which is known to mediate the ubiquitination of interacting targets. The deficiency of FBW2 increased the abundance of AGO1. In contrast, overexpression of FBW2 significantly decreased the protein level of AGO1, demonstrating the negative function of FBW2 in AGO1 stability. However, the reduced level of AGO1 in the transgenic plants was not fully restored following MG132 treatment (Earley et al., 2010). These results indicated that AGO1 destabilization by either PO or FBW2 is not subjected to the Ubiquitin-proteasome system (UPS) pathway. Indeed, AGO1 degradation was mediated by autophagy, which initiates the orderly degradation and recycling of cellular components. AGO1 protein dramatically accumulated in response to treatment with 3-MA, an autophagy flux inhibitor or E64, an autophagic protease inhibitor. Furthermore, AGO1 co-localized with autophagosomal membrane protein ATG8, strongly indicating that AGO1 was associated with the autophagosome (Derrien et al., 2012). Although autophagy has been considered to represent a random method of degradation, many recent studies have shown that specific ubiquitin E3 ligases and substrate ubiquitination are tightly linked to the autophagy pathway to remove aggregated proteins (Kraft et al., 2010; Myeku and Figueiredo-Pereira, 2011). In this context, autophagy-dependent degradation of ubiquitinated AGO1 in connection with endogenous SCF E3 ligase complexes can be understood (Fig. 1). However, this autophagy-dependent pathway may not always occur in other viral suppressors. For instance, when MG132 was infiltrated into $N$. benthamiana leaves $2 \mathrm{~h}$ before co-infiltration with Agrobacterium strains to express P25 and AGO1, the accumulation of AGO1 increase $\sim 10$-fold even in the presence of the P25 suppressor, implying the indirect or direct involvement of the UPS system in AGO1 destabilization (Chiu et al., 2010).

In fact, two pathways for AGO1 destabilization, autophagic and UPS pathways, could function independently in plants. For instance, if two viral F-box proteins, P0 and P25, target different negative regulators of $A G O 1$, assuming that at least two negative regulators of AGO1 exist, the different mechanisms could be understood. However, the presence of two independent and pathogen-specific pathways for AGO1 degradation should be further demonstrated experimentally using specific pathway inhibitors and mutants (Fig. 1).

\section{POST-TRANSLATIONAL REGULATION OF HYL1 AND DRB4}

There are four DCLs in Arabidopsis. DCL1 processes pri- 


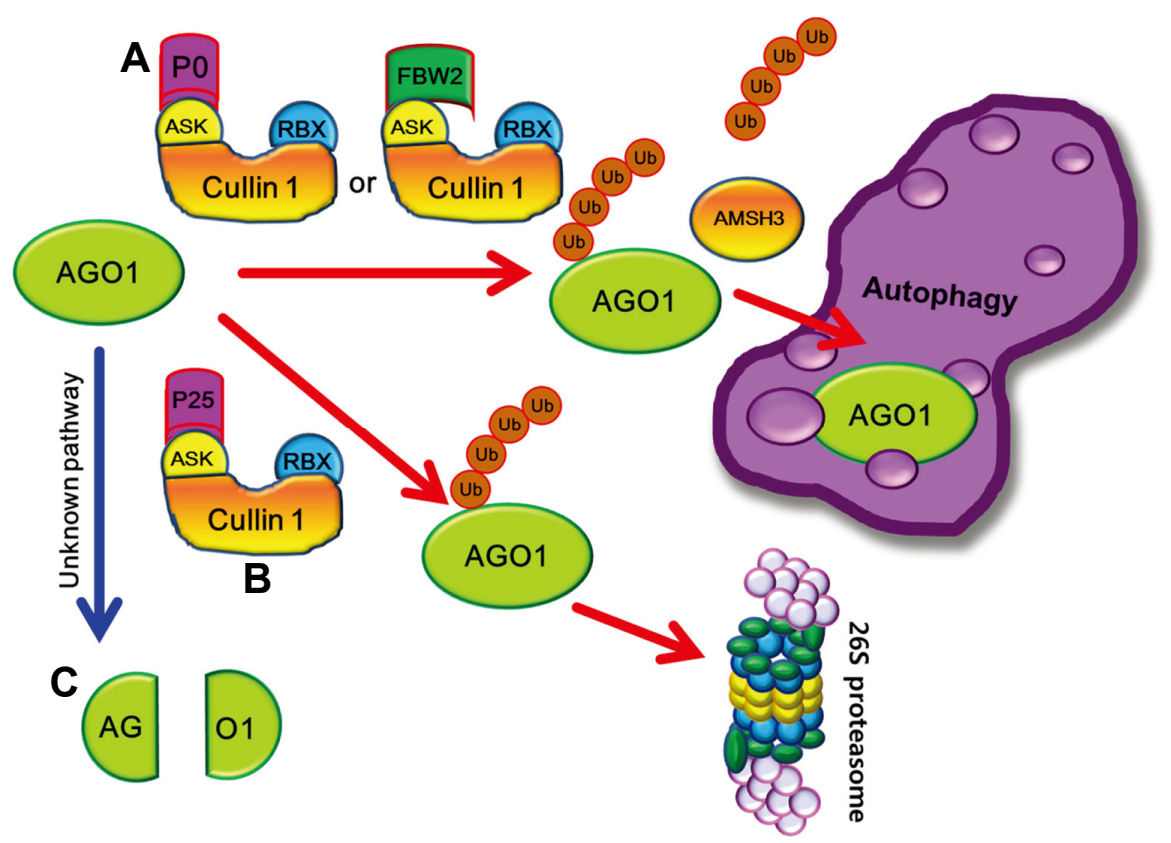

Fig. 1. A model for the proteolytic regulation of AGO1. (A) Viral P0 or endogenous F-box protein FBW2 mediate AGO1 destabilization. PO protein hijacks the host SCF-complex to modify AGO1 and is then subjected to autophagic degradation following deubiquitination. FBW2 may perform a similar process through the SCF complex. (B) Viral P25 suppressor mediated AGO1 destabilization. In contrast to the role of P0, P25 seems to guide the ubiquitinated $\mathrm{AGO} 1$ into the $26 \mathrm{~S}$ proteasome. (C) AGO1 is specifically cleaved into a $\sim 45-\mathrm{kDa}$ polypeptide with or without viral suppressors. Thus, an unknown cleavage pathway may exist to turn over AGO1 activity.
miRNA into miRNAs, DCL2 processes double-stranded RNA into 22-nucleotide small interfering RNAs and is redundantly involved in miRNA processing. DCL3 produces endogenous RDR2-dependent siRNAs for DNA methylation. DCL4 is responsible for the production of endogenous RDR6-dependent trans-acting siRNAs (tasiRNAs) (Gasciolli et al., 2005; Moissiard and Voinnet, 2006; Xie et al., 2004). Arabidopsis encodes five double-stranded RNA binding (DRB) proteins (Eamens et al., 2012). By assisting DCL1, DRB1 and DRB2 are involved in miRNA biogenesis. As a partner of DCL4, DRB4 functions in small interfering RNA (siRNA) pathways (Curtin et al., 2008). Together with DCL4, DRB4 is responsible for the production of TAS3 trans-acting siRNAs and in the generation of antiviral responses (Adenot et al., 2006; Fukudome et al., 2011; Hiraguri et al., 2005; Jakubiec et al., 2012; Nakazawa et al., 2007). DRB3 is involved in methylation-mediated antiviral defense with DCL3 and AGO4 (Raja et al., 2014). Despite their important roles in gene expression, epigenetic control, and defense, the post-translational regulation of these DCL proteins and their associated proteins have not been reported until recently, except DRB1, which is also known as HYL1, and DRB4.

HYL1 is a double-stranded RNA-binding protein. hyl1-2 mutants displayed pleiotropic developmental disorders, including shorter stature, delayed flowering, leaf upward curling, partial infertility, defective apical shoot, and root growth (Lu and Fedoroff, 2000). HYL1 forms a complex with DCL1 and SERRATE to increase the accuracy of pri-miRNA processing into mature miRNAs. Structural analysis demonstrated that RNA-binding domain 1 recognizes the miRNA/miRNA* region of the precursor as a dimer, mediated by RNA-binding domain 2 (Yang et al., 2010). Furthermore, the homo-dimerization of HYL1 is very important for adjusting the cleavage site in pri-miRNAs. The activity of HYL1 is also crucial to select the guide strand of miRNAs upon AGO1 loading (Wu et al., 2013). HYL1 requires C-TERMINAL DOMAIN PHOSPHATASE-LIKE 1 (CPL1), which dephosphorylates HYL1 proteins, for optimal activity (Manavel- la et al., 2012). That study showed, for the first time, that the post-translational modification of HYL1, involving the removal of a phosphate group, is important for miRNA processing accuracy and strand selection.

Recently, using a DNA/silver nanocluster sensor-based screening method, Cho et al. (2014) identified a new component in miRNA biogenesis (Shah et al., 2012; 2014; 2015; Yang and Vosch, 2011). Interestingly, the isolated component was defined as constitutive photomorphogenic 1 (COP1), which plays a central role in photomorphogenesis. miRNA analyses have shown that COP1 also has an essential role in miRNA biogenesis. COP1 led to the reduction of HYL1 and subsequently compromised miRNA biogenesis. The role of COP1 in maintaining HYL1 expression was shown to be tightly associated with post-translational regulation. Levels of HYL1 were clearly increased by MG132 but not by the other specific proteasome inhibitors, clasto-lactacystin $\beta$-lactone and epoxomicin. Furthermore, HYL1 destabilization was not blocked by PRY-41, an inhibitor of E1 enzyme for the ubiquitin-conjugating process, implying that UPS is not responsible for HYL1 degradation. On the other hand, the possible involvement of autophagy was investigated using an autophagy inhibitor, 3-MA, and an activator, BTH. As a result, HYL1 abundance was also shown as not modulated by autophagy. Taken together, these results showed that neither the UPS pathway nor autophagy is responsible for the degradation of HYL1. Interestingly, HYL1 was specifically cleaved into a $\sim 26-\mathrm{kDa} \mathrm{N}$-terminal fragment by a crude cytoplasmic extract in vitro. HYL1 cleavage was efficiently blocked by either MG132 or E64 in vitro. Consistently, the cytoplasmic accumulation of HYL1 has been clearly demonstrated following the treatment of HYL1-CFP transgenic seedlings with MG132. Based on those results, it was suggested that HYL1 may be cleaved by an as yet unknown protease existing in the cytoplasm. More interestingly, Cho et al. showed that HYL1 degradation is regulated by the light-to-dark transition. During the daytime, COP1 moves to the cytoplasm stabilizing HYL1, 


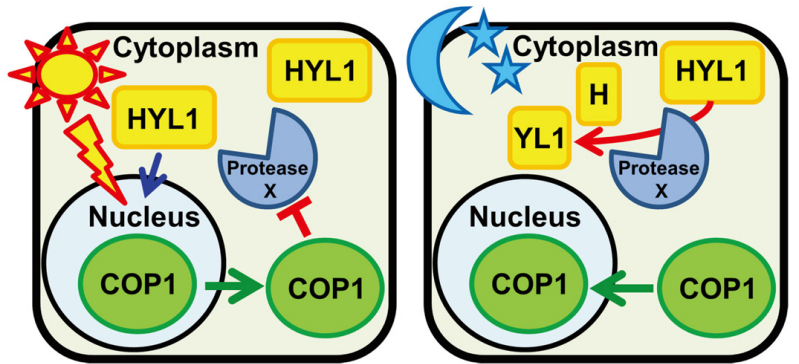

Fig. 2. A model of the proteolytic regulation of HYL1 for miRNA homeostasis. During the day, COP1 moves to the cytoplasm and suppresses the cleavage of HYL1 by protease X. COP1 remobilizes into the nucleus liberating the protease $X$ at night. Non-canonical role of COP1, which is unrelated to the UPS pathway, may be responsible for the suppression of protease $\mathrm{X}$.

possibly by inhibiting the protease. In addition, during the night, COP1 migrates into the nucleus and removes the protease activity required for HYL1 degradation. Those results indicated that light signaling is integrated into miRNA biogenesis. Although the protease involved in HYL1 cleavage was not clarified, the study primarily showed that the presence of a third proteolytic regulatory console, distinct to both the UPS pathway and autophagy (Fig. 2).

Marrocco et al. (2012). found that DRB4 protein directly interacts with APC10/DCO1, a subunit of anaphase promoting complex (APC/C), or cyclosome, a cell cycle-regulated ubiquitin ligase that controls progression of the cell cycle. The dsRNA binding domain 2 of DRB4 was required for its homodimerization and for the interactions with APC10 and DCL4. Moreover, the level of DRB4 was dramatically increased in response to MG132 treatment and in APC10 RNAi knockdown mutants, implying that DRB4 might be a target of the APC complexmediated UPS pathway. However, to confirm the involvement of UPS in the proteolytic regulation of DRB4, further detailed investigations are needed. These should aim to 1) investigate the ubiquitination of DRB4 in vivo and in vitro by the APC complex, 2) test specific proteasome inhibitors such as clastolactacystin $\beta$-lactone and epoxomicin, and 3) test the possible involvement of autophagy.

\section{DISCUSSION}

In plants, several studies have shed light on the proteolytic regulation of microprocessor components and RNA silencing complexes. In the context of virus-host interactions, the destabilization of AGO proteins has been discussed. AGO1 protein seems to be regulated either by SCF complex-mediated autophagy or by the UPS pathway according to the type of infecting viruses (Baumberger et al., 2007; Bortolamiol et al., 2007; Chiu et al., 2010; Derrien et al., 2012; Fusaro et al., 2012). Although the degradations of AGO2, AGO4, AGO5, AGO6, and AGO9 have also been monitored using transient expression systems, the mechanism of degradation has not been fully elucidated. Notably, the level of AGO1 protein was significantly diminished in all mutants in which the miRNA biogenesis was affected (Derrien et al., 2012). Reduced levels of AGO1 protein in hen $1-1$ seedlings were restored by E64d treatment, implying the involvement of autophagy. When GFP-AGO1 was coexpressed with RFP-ATG8a fusion protein in tobacco cells,
GFP-AGO1 associated with RFP-ATG8a within small intracellular structures like acid vascular organelles (AVO). The colocalization was further recapitulated by a co-immunoprecipitation assay in planta, strongly suggesting that AGO1 associates with ATG8 in the autophagosome. Based on those results, Derrian et al. (2012) suggested that the proteolytic regulation of AGO1 in the context of non-viral infection may also be attributed to SCF complex-mediated autophagy. However, the detailed mechanism of AGO1 destabilization needs to be further established considering the possible involvement of an unknown pathway for AGO1 degradation. We propose this hypothesis based in the analysis of P0-induced AGO1 degradation (Baumberger et al., 2007). Regardless of whether P0 was expressed, AGO1 was cleaved into a 45-kDa polypeptide corresponding to the N-terminal of AGO1, and that fragment was increased when treated with MG132. Hence, Baumberger et al. (2007) suggested that the $\sim 45-\mathrm{kDa}$ protein is evidence of an AGO1 degradation pathway that is not strictly dependent on P0. If AGO1 is solely degraded by autophagy or the $26 \mathrm{~S}$ proteasome, the specific cleavage pattern of AGO1 could not be monitored, indicating that an unknown pathway may exist. Autophagy involves the digestion of target proteins and the disassembly of polyubiquitinated proteins into short peptide fragments by the $26 \mathrm{~S}$ proteasome. Although in some cases, the $26 \mathrm{~S}$ proteasome produces biologically active and functional molecules such as antigenic or signaling polypeptides (Rock and Goldberg, 1999), the $45-\mathrm{kDa}$ polypeptide is too large to consider it a product of the $26 \mathrm{~S}$ proteasome. Likewise, HYL1 is specifically cleaved into a $\sim 25-\mathrm{kDa}$ polypeptide at the $\mathrm{N}$ terminal region by an as yet unknown cytoplasmic protease, which seems to be be not included in the acid compartments because the catalytic activity of the protease is maintained even under neutral $\mathrm{pH}$ (Cho et al., 2014). Therefore, we suggest that the cleavage of AGO1 by non-AVO cytoplasmic proteases cannot be ruled out, at least in plants. Even though AGO1 and HYL1 might be targets of the unidentified cytoplasmic protease or class of proteases, the detailed mechanisms underlying their degradation could be distinct. Because the protein levels of HYL1 and AGO1 are differentially modulated by chemical inhibitors such as MG132 and 3-MA, and the half-life of HYL1 (less than $2 \mathrm{~h}$ ) is much shorter than that of AGO1 (more than one day) (Baumberger et al., 2007; Cho et al., 2014; Derrien et al., 2012). The pathogen-induced degradation of AGO1 is very important for studying plant defense systems. By blocking the major RNA-silencing mechanism, viruses are able to escape from the RNA interference-based plant defense system and efficiently spread within host plants. Therefore, by obtaining detailed knowledge on the proteolytic regulation of AGO1, plant defense systems can be manipulated to confer high viral resistance.

Furthermore, the proteolytic regulation of HYL1 by the lightdark transition must be further investigated in relation to the light signaling pathway. The developmental transition between skotomorphogenesis and photomorphogenesis is essential in the early development of plants, and this has been widely studied in terms of the proteolytic regulation of light receptors and transcription factors. The integration of light signaling into miRNA biogenesis, per se, has been scarcely addressed. Thus, future studies investigating the crosstalk between two major regulatory pathways, light signaling and miRNA biogenesis, could be essential to fully delineate the early development of plants. Moreover, to date, the proteolytic regulation of the core microprocessor, DCL1 and SE, has not been addressed in plants. Hence, to fully understand miRNA biogenesis in plants, 
investigations on the proteolytic pathways of DCL1 and SE are envisaged as future challenges.

\section{ACKNOWLEDGMENTS}

This work was supported under the framework of an international cooperation program managed by the National Research Foundation of Korea (2015K2A1A2071052).

\section{REFERENCES}

Adenot, X., Elmayan, T., Lauressergues, D., Boutet, S., Bouche, N., Gasciolli, V., and Vaucheret, H. (2006). DRB4-dependent TAS3 trans-acting siRNAs control leaf morphology through AGO7. Curr. Biol. 16, 927-932.

Alvarado, V.Y., and Scholthof, H.B. (2011). AGO2, A new argonaute compromising plant virus accumulation. Front Plant Sci. 2, 112.

Bartel, D.P. (2004). MicroRNAs, genomics, biogenesis, mechanism, and function. Cell 116, 281-297.

Baulcombe, D. (2004). RNA silencing in plants. Nature 431, 356363.

Baumberger, N., Tsai, C.H., Lie, M., Havecker, E., and Baulcombe, D.C. (2007). The Polerovirus silencing suppressor PO targets ARGONAUTE proteins for degradation. Curr. Biol. 17, 1609-1614.

Ben Chaabane, S., Liu, R., Chinnusamy, V., Kwon, Y., Park, J.H., Kim, S.Y., Zhu, J.K., Yang, S.W., and Lee, B.H. (2013). STA1, an Arabidopsis pre-mRNA processing factor 6 homolog, is a new player involved in miRNA biogenesis. Nucleic Acids Res. 41, 1984-1997.

Bielewicz, D., Kalak, M., Kalyna, M., Windels, D., Barta, A., Vazquez, F., Szweykowska-Kulinska, Z., and Jarmolowski, A. (2013). Introns of plant pri-miRNAs enhance miRNA biogenesis. EMBO Rep. 14, 622-628.

Bohmert, K., Camus, I., Bellini, C., Bouchez, D., Caboche, M., and Benning, C. (1998). AGO1 defines a novel locus of Arabidopsis controlling leaf development. EMBO J. 17, 170-180.

Bortolamiol, D., Pazhouhandeh, M., Marrocco, K., Genschik, P., and Ziegler-Graff, V. (2007). The Polerovirus $F$ box protein PO targets ARGONAUTE1 to suppress RNA silencing. Curr. Biol. 17, 1615-1621.

Brodersen, P., and Voinnet, O. (2009). Revisiting the principles of microRNA target recognition and mode of action. Nat. Rev. Mol. Cell Biol. 10, 141-148.

Callis, J. (2014). The ubiquitination machinery of the ubiquitin system. Arabidopsis Book 12, e0174.

Cardozo, T., and Pagano, M. (2004). The SCF ubiquitin ligase, insights into a molecular machine. Nat. Rev. Mol. Cell Biol. 5, 739-751.

Chiu, M.H., Chen, I.H., Baulcombe, D.C., and Tsai, C.H. (2010). The silencing suppressor P25 of Potato virus $\mathrm{X}$ interacts with Argonaute 1 and mediates its degradation through the proteasome pathway. Mol. Plant Pathol. 11, 641-649.

Cho, S.K., Ben Chaabane, S., Shah, P., Poulsen, C.P., and Yang, S.W. (2014). COP1 E3 ligase protects HYL1 to retain microRNA biogenesis. Nat. Commun. 5, 5867.

Curtin, S.J., Watson, J.M., Smith, N.A., Eamens, A.L., Blanchard, C.L., and Waterhouse, P.M. (2008). The roles of plant dsRNAbinding proteins in RNAi-like pathways. FEBS Lett. 582, 27532760.

Denli, A.M., Tops, B.B., Plasterk, R.H., Ketting, R.F., and Hannon, G.J. (2004). Processing of primary microRNAs by the Microprocessor complex. Nature 432, 231-235.

Derrien, B., Baumberger, N., Schepetilnikov, M., Viotti, C., De Cillia J., Ziegler-Graff, V., Isono, E., Schumacher, K., and Genschik, P. (2012). Degradation of the antiviral component ARGONAUTE1 by the autophagy pathway. Proc. Natl. Acad. Sci. USA 109, $15942-15946$

Dong, Z., Han, M.H., and Fedoroff, N. (2008). The RNA-binding proteins HYL1 and SE promote accurate in vitro processing of pri-miRNA by DCL1. Proc. Natl. Acad. Sci. USA 105, 9970-9975

Eamens, A.L., Wook Kim, K., and Waterhouse, P.M. (2012). DRB2, DRB3 and DRB5 function in a non-canonical microRNA pathway in Arabidopsis thaliana. Plant Signal. Behav. 7, 1224-1229.

Earley, K., Smith, M., Weber, R., Gregory, B., and Poethig, R.
(2010). An endogenous F-box protein regulates ARGONAUTE1 in Arabidopsis thaliana. Silence 1, 15.

Fukudome, A., Kanaya, A., Egami, M., Nakazawa, Y., Hiraguri, A., Moriyama, H., and Fukuhara, T. (2011). Specific requirement of DRB4, a dsRNA-binding protein, for the in vitro dsRNA-cleaving activity of Arabidopsis Dicer-like 4. RNA 17, 750-760.

Fusaro, A.F., Correa, R.L., Nakasugi, K., Jackson, C., Kawchuk, L., Vaslin, M.F., and Waterhouse, P.M. (2012). The Enamovirus PO protein is a silencing suppressor which inhibits local and systemic RNA silencing through AGO1 degradation. Virology 426, 178-187.

Gasciolli, V., Mallory, A.C., Bartel, D.P., and Vaucheret, H. (2005). Partially redundant functions of Arabidopsis DICER-like enzymes and a role for DCL4 in producing trans-acting siRNAs. Curr. Biol. 15, 1494-1500.

Gu, S., Jin, L., Zhang, Y., Huang, Y., Zhang, F., Valdmanis, P.N., and Kay, M.A. (2012). The loop position of shRNAs and premiRNAs is critical for the accuracy of dicer processing in vivo. Cell 151, 900-911.

Hare, P.D., Seo, H.S., Yang, J.Y., and Chua, N.H. (2003) Modulation of sensitivity and selectivity in plant signaling by proteasomal destabilization. Curr. Opin. Plant Biol. 6, 453-462.

Hiraguri, A., Itoh, R., Kondo, N., Nomura, Y., Aizawa, D., Murai, Y., Koiwa, H., Seki, M., Shinozaki, K., and Fukuhara, T. (2005). Specific interactions between Dicer-like proteins and HYL1/DRBfamily dsRNA-binding proteins in Arabidopsis thaliana. Plant Mol. Biol. 57, 173-188.

Jakubiec, A., Yang, S.W., Chua, N.H. (2012). Arabidopsis DRB4 protein in antiviral defense against Turnip yellow mosaic virus infection. Plant J. 69, 14-25.

Kim, V.N. (2005). MicroRNA biogenesis, coordinated cropping and dicing. Nat. Rev. Mol. Cell Biol. 6, 376-385.

Kraft, C., Peter, M., and Hofmann, K. (2010). Selective autophagy, ubiquitin-mediated recognition and beyond. Nat. Cell Biol. 12, 836-841.

Kurihara, Y., Takashi, Y., and Watanabe, Y. (2006). The interaction between DCL1 and HYL1 is important for efficient and precise processing of pri-miRNA in plant microRNA biogenesis. RNA 12, 206-212.

Kwon, S.C., Nguyen, T.A., Choi, Y.G., Jo, M.H., Hohng, S., Kim, V.N., and Woo, J.S. (2016). Structure of human DROSHA. Cell 164, 81-90.

Landthaler, M., Yalcin, A., and Tuschl, T. (2004). The human DiGeorge syndrome critical region gene 8 and Its D. melanogaster homolog are required for miRNA biogenesis. Curr. Biol. 14, 21622167.

Lu, C., and Fedoroff, N. (2000). A mutation in the Arabidopsis HYL1 gene encoding a dsRNA binding protein affects responses to abscisic acid, auxin, and cytokinin. Plant Cell 12, 2351-2366.

Manavella, P.A., Hagmann, J., Ott, F., Laubinger, S., Franz, M., Macek, B., and Weigel, D. (2012). Fast-forward genetics identifies plant $\mathrm{CPL}$ phosphatases as regulators of miRNA processing factor HYL1. Cell 151, 859-870.

Meng, Y., Shao, C., Ma, X., and Wang, H. (2013). Introns targeted by plant microRNAs, a possible novel mechanism of gene regulation. Rice (NY) 6, 8.

Moissiard, G., and Voinnet, O. (2006). RNA silencing of host transcripts by cauliflower mosaic virus requires coordinated action of the four Arabidopsis Dicer-like proteins. Proc. Natl. Acad. Sci. USA 103, 19593-19598.

Myeku, N., and Figueiredo-Pereira, M.E. (2011). Dynamics of the degradation of ubiquitinated proteins by proteasomes and autophagy, association with sequestosome 1/p62. J. Biol. Chem. 286, 22426-22440.

Nakazawa, Y., Hiraguri, A., Moriyama, H., and Fukuhara, T. (2007). The dsRNA-binding protein DRB4 interacts with the Dicer-like protein DCL4 in vivo and functions in the trans-acting siRNA pathway. Plant Mol. Biol. 63, 777-785

Nguyen, T.A., Jo, M.H., Choi, Y.G., Park, J., Kwon, S.C., Hohng, S., Kim, V.N., and Woo, J.S. (2015). Functional anatomy of the human microprocessor. Cell 161, 1374-1387

Okamura, K., Hagen, J.W., Duan, H., Tyler, D.M., and Lai, E.C. (2007). The mirtron pathway generates microRNA-class regulatory RNAs in Drosophila. Cell 130, 89-100.

Parizotto, E.A., Dunoyer, P., Rahm, N., Himber, C., and Voinnet, O. (2004). In vivo investigation of the transcription, processing, 
endonucleolytic activity, and functional relevance of the spatial distribution of a plant miRNA. Genes Dev. 18, 2237-2242.

Park, W., Li, J., Song, R., Messing, J., and Chen, X. (2002). CARPEL FACTORY, a Dicer homolog, and HEN1, a novel protein, act in microRNA metabolism in Arabidopsis thaliana. Curr. Biol. 12, 1484-1495

Pazhouhandeh, M., Dieterle, M., Marrocco, K., Lechner, E., Berry, B., Brault, V., Hemmer, O., Kretsch, T., Richards, K.E., Genschik, P., and Ziegler-Graff, V. (2006). F-box-like domain in the polerovirus protein $\mathrm{PO}$ is required for silencing suppressor function. Proc. Natl. Acad. Sci. USA 103, 1994-1999.

Pfeffer, S., Dunoyer, P., Heim, F., Richards, K.E., Jonard, G., and Ziegler-Graff, V. (2002). PO of beet Western yellows virus is a suppressor of posttranscriptional gene silencing. J. Virol. 76, 6815-6824.

Raja, P., Jackel, J.N., Li, S., Heard, I.M., and Bisaro, D.M. (2014). Arabidopsis double-stranded RNA binding protein DRB3 participates in methylation-mediated defense against geminiviruses. J. Virol. 88, 2611-2622.

Ren, G., and Yu, B. (2012). Critical roles of RNA-binding proteins in miRNA biogenesis in Arabidopsis. RNA Biol. 9, 1424-1428.

Rock, K.L., and Goldberg, A.L. (1999). Degradation of cell proteins and the generation of MHC class I-presented peptides. Annu. Rev. Immunol. 17, 739-779.

Rogers K, and Chen X (2013). Biogenesis, turnover, and mode of action of plant microRNAs. Plant Cell 25, 2383-2399.

Saito, K., Ishizuka, A., Siomi, H., and Siomi, M.C. (2005). Processing of pre-microRNAs by the Dicer-1-Loquacious complex in Drosophila cells. PLoS Biol. 3, e235.

Shah, P., Rorvig-Lund, A., Chaabane, S.B., Thulstrup, P.W., Kjaergaard, H.G., Fron, E., Hofkens, J., Yang, S.W., and Vosch, T. (2012). Design aspects of bright red emissive silver nanoclusters/DNA probes for microRNA detection. ACS Nano 6, 8803-8814.

Shah, P., Thulstrup, P.W., Cho, S.K., Bjerrum, M.J., and Yang, S.W. (2014) DNA-RNA chimera indicates the flexibility of the backbone influences the encapsulation of fluorescent AgNC emitters. Chem. Commun. 50, 13592-13595.

Shah, P., Choi, S.W., Kim, H.J., Cho, S.K., Thulstrup, P.W., Bjerrum, M.J., Bhang, Y.J., Ahn, J.C., and Yang, S.W. (2015). DNA/RNA chimera templates improve the emission intensity and target the accessibility of silver nanocluster-based sensors for human microRNA detection. Analyst 140, 3422-3430.

Speth, C., Willing, E.M., Rausch, S., Schneeberger, K., and Laubinger, S. (2013). RACK1 scaffold proteins influence miRNA abundance in Arabidopsis. Plant J. 76, 433-445.

Valencia-Sanchez, M.A., Liu, J., Hannon, G.J., and Parker, R. (2006). Control of translation and mRNA degradation by miRNAs and siRNAs. Genes Dev. 20, 515-524.

Vaucheret, H., Vazquez, F., Crete, P., and Bartel, D.P. (2004). The action of ARGONAUTE1 in the miRNA pathway and its regulation by the miRNA pathway are crucial for plant development. Genes Dev. 18, 1187-1197.

Vaucheret, H., Mallory, A.C., and Bartel, D.P. (2006) AGO1 homeostasis entails coexpression of MIR168 and AGO1 and preferential stabilization of miR168 by AGO1. Mol. Cell 22, 129136.

Voinnet, O. (2005). Induction and suppression of RNA silencing, insights from viral infections. Nat. Rev. Genet. 6, 206-220.

Voinnet, O. (2009). Origin, biogenesis, and activity of plant microRNAs. Cell 136, 669-687.

von Arnim, A.G. (2001). A hitchhiker's guide to the proteasome. Sci STKE 2001, pe2.

Wu, X., Shi, Y., Li, J., Xu, L., Fang, Y., Li, X., and Qi, Y. (2013). A role for the RNA-binding protein MOS2 in microRNA maturation in Arabidopsis. Cell Res. 23, 645-657.

Xie, Z., Johansen, L.K., Gustafson, A.M., Kasschau, K.D., Lellis, A.D., Zilberman, D., Jacobsen, S.E., and Carrington, J.C. (2004) Genetic and functional diversification of small RNA pathways in plants. PLoS Biol. 2, E104.

Yang, S.W., and Vosch, T. (2011). Rapid detection of microRNA by a silver nanocluster DNA probe. Anal Chem 83, 6935-6939.

Yang, S.W., Chen, H.Y., Yang, J., Machida, S., Chua, N.H., and Yuan, Y.A. (2010). Structure of Arabidopsis HYPONASTIC LEAVES1 and its molecular implications for miRNA processing Structure 18, 594-605.

Zeng, Y., Yi, R., and Cullen, B.R. (2005). Recognition and cleavage of primary microRNA precursors by the nuclear processing enzyme Drosha. EMBO J. 24, 138-148.

Zhan, X., Wang, B., Li, H., Liu, R., Kalia, R.K., Zhu, J.K., and Chinnusamy, V. (2012). Arabidopsis proline-rich protein important for development and abiotic stress tolerance is involved in microRNA biogenesis. Proc. Natl. Acad. Sci. USA 109, 1819818203

Zhang, X., Yuan, Y.R., Pei, Y., Lin, S.S., Tuschl, T., Patel, D.J., and Chua, N.H. (2006). Cucumber mosaic virus-encoded 2b suppressor inhibits Arabidopsis Argonaute 1 cleavage activity to counter plant defense. Genes Dev. 20, 3255-3268.

Zhang, S., Xie, M., Ren, G., and Yu, B. (2013). CDC5, a DNA binding protein, positively regulates posttranscriptional processing and/or transcription of primary microRNA transcripts. Proc. Natl. Acad. Sci. USA 110, 17588-17593.

Zhang, S., Liu, Y., and Yu, B. (2014). PRL1, an RNA-binding protein, positively regulates the accumulation of miRNAs and siRNAs in Arabidopsis. PLoS Genet. 10, e1004841. 